\title{
A Novel Radiofrequency Device to Monitor Changes in Pulmonary Fluid in Dialysis Patients
}

This article was published in the following Dove Press journal:

Medical Devices: Evidence and Research

Jeffrey J Connaire'

Matthew L Sundermann ${ }^{2}$

Ramu Perumal ${ }^{2}$

Charles A Herzog ${ }^{3}$

'Davita Clinical Research, Minneapolis, MN, USA; ${ }^{2}$ ZOLL, Pittsburgh, PA, USA;

${ }^{3}$ Cardiology Division, Department of Internal Medicine, Hennepin Healthcare/ University of Minnesota, Minneapolis, MN, USA
Correspondence: Jeffrey J Connaire DaVita Clinical Research, $825 \mathrm{~S} 8^{\text {th }} \mathrm{St}$, Suite 300, Minneapolis, MN 55404, USA

$\mathrm{Tel}+|6| 2202-|58|$

$\mathrm{Fax}+|866852-324|$

Email Jeffrey.Connaire@davita.com
Background and Objectives: Fluid monitoring is an important management strategy in patients with chronic kidney disease (CKD) and heart failure (HF). The $\mu$ Cor $^{\mathrm{TM}}$ Heart Failure and Arrhythmia Management System uses a radiofrequency-based thoracic fluid index (TFI) to track pulmonary edema. During hemodialysis, the acute removal of fluid through ultrafiltration offers a model for measuring a patient's fluid status. The objective of the study was to assess the relationship between the device measured TFI and ultrafiltration volume (UFV). Design, Setting, Participants, and Measurements: Patients undergoing chronic dialysis with and without heart failure were enrolled in the study. The relationship between TFI and UFV in each individual subject was assessed by calculating the Pearson correlation coefficient $(r)$. The average correlation across all subjects was calculated through the use of the Fisher's $z$ transform. Responder analysis was performed to assess the magnitude of change in TFI before and after dialysis.

Results: Twenty subjects were enrolled in the trial. The mean volume of fluid removal was $3.63 \mathrm{~L}$ (SD $0.88 \mathrm{~L}$ ). The mean correlation based on Fisher's transform was $0.95 \mathrm{CI}$ $(0.92-0.99)$. Responder analysis showed that the mean reduction of TFI after dialysis was $5.5 \% \pm 3.8$.

Conclusion: The $\mu$ Cor system provides radiofrequency-based measurements of thoracic fluid which correlate well with total body fluid removal in a real-world setting. Fluid management based on the radar-derived TFI may provide benefits to dialysis patients and serves as a potential model for pulmonary edema common to the clinical course of heart failure.

Keywords: heart failure, total body fluid monitoring, dialysis, wearables, ultrafiltration

\section{Introduction}

Fluid monitoring is an important management strategy in patients with chronic kidney disease (CKD) and heart failure (HF). ${ }^{1,2}$ Assessment of fluid status changes in these patients is especially important when introducing therapies that target diuresis or fluid removal, and serial fluid measurements are often required to allow for non-invasive fluid monitoring to capture temporal trends. Symptomatic response to fluid removal in patients with both CKD and HF presents a unique classification challenge where fluid assessment before and after ultrafiltration (UFV) offers context to the underlying disease. ${ }^{3}$ Furthermore, measurement of lung fluid using radiofrequency $(\mathrm{RF})$ measurements may serve as a viable option to remotely monitor patients with heart failure, and RF when delivered at the optimum frequency range offers fluid detection capabilities in the lungs. ${ }^{4}$ Detection of early fluid changes in CKD and HF patients may potentially assist with remote patient management. 
Wearable sensors are promising tools for fluid monitoring and have the potential to improve patient outcomes. ${ }^{5}$ Recent studies of fluid monitoring in the clinical setting have involved point-of-care approaches such as lung ultrasonography (LUS) or device measured bioimpedance to aid in the diagnosis and prognosis of fluid accumulation. ${ }^{6,7}$ A wearable sensor capable of taking serial RF measurements would be a valuable tool for the clinician as fluid status could then be assessed through temporal trends in RF measurements.

Clinically, serial fluid status evaluation is most often required when rapid changes in fluid occur in settings such as hemodialysis, as well as in clinical settings such as heart failure where pulmonary edema worsens over time due to acute disease exacerbations. Fluid removal during hemodialysis offers a unique opportunity to assess acute fluid changes since a liter or more of fluid is removed over a short period of time. Furthermore, a previous study of 75 hemodialysis patients demonstrated that $63 \%$ of the study's subjects were shown to have moderate to severe lung congestion before UFV began. ${ }^{8}$

In this study, the performance of the $\mu$ Cor system, a non-invasive wearable monitor that assesses fluid change through RF measurements, was evaluated in the context of hemodialysis. The $\mu$ Cor system consists of a patch-based sensor and is designed to non-invasively record, store, and transmit physiological data to medical professionals. The $\mu$ Cor system records an RF-derived measurement termed thoracic fluid index (TFI), electrocardiogram, heart rate, respiration rate, activity, and posture. Measurements acquired by the $\mu$ Cor system are transmitted wirelessly to a remote server with dedicated software for processing.

The primary objective of the study was to assess the correlation of TFI measurements taken by the $\mu$ Cor system to fluid removal within dialysis patients. Our hypothesis was that the $\mu$ Cor system would accurately track total body fluid removal with RF measurements in dialysis subjects.

\section{Materials and Methods}

The study was designed as a prospective and nonsignificant risk study. Subjects were informed of the purpose of the trial, were required to provide signed informed consent prior to any study-related procedures, and were informed that the trial was conducted in accordance with the Declaration of Helsinki. The study was IRB approved (IntegReview). Subjects included in the study had to be at least 21 years of age, had to be scheduled to undergo hemodialysis 3 times a week, had to have had ongoing hemodialysis for at least 3 months, and had to have been prescribed a net fluid removal of at least $2.5 \mathrm{~L}$. The trial protocol and summary results are available under NCT03072732 at ClinicalTrials.gov, and the authors have direct access to the primary data from the trial. Additional data requests will not be granted due to the proprietary nature of the study device. The amount of fluid removal on the day of each test was determined per standard of care. Subjects underwent one hemodialysis session at one of the three separate hemodialysis sites.

\section{$\mu$ Cor System}

The study device shown in Figure 1, commercially available as the $\mu \mathrm{Cor}^{\mathrm{TM}}$ Heart Failure and Arrhythmia Management System, is an adhesive wearable sensor that uses RF reflections to establish TFI. The device's antenna emits RF signals which propagate through the lungs where signals are then reflected back to the device. Changes in radar wave signal path duration and strength indicate changes in fluid accumulation; the waves are highly modulated by tissue hydration. RF reflections are then used to calculate TFI, a relative index that is normalized to a baseline. Valid TFI measurements are distinguished from invalid TFI measurements through device thresholds including activity level measured through an accelerometer on the device sensor, RF signal quality, and sensor position. The accelerometer is set so that TFI measurements are not taken during a maximum threshold of activity as well as within specific pitch and roll ranges. The TFI validity requirements ensure that readings are taken during consistent conditions. The $\mu$ Cor system's intended use is in outpatient clinics and home settings.

\section{Study Procedure}

At the clinical baseline, the subject's medical history, weight, blood pressure, and heart rate were recorded. Subjects were placed in the dialysis chair per standard of care throughout the duration of the study. To optimize measurements, subjects were instructed to maintain the same posture throughout fluid removal as much as possible.

RF readings were recorded during dialysis with the $\mu$ Cor device placed in the left midaxillary position. The $\mu$ Cor device was programmed to record measurements every $3 \mathrm{~min}( \pm 1 \mathrm{~min})$ during the hemodialysis session, including at least $15 \mathrm{~min}$ before the start of the hemodialysis session through at least $15 \mathrm{~min}$ after the end of the 


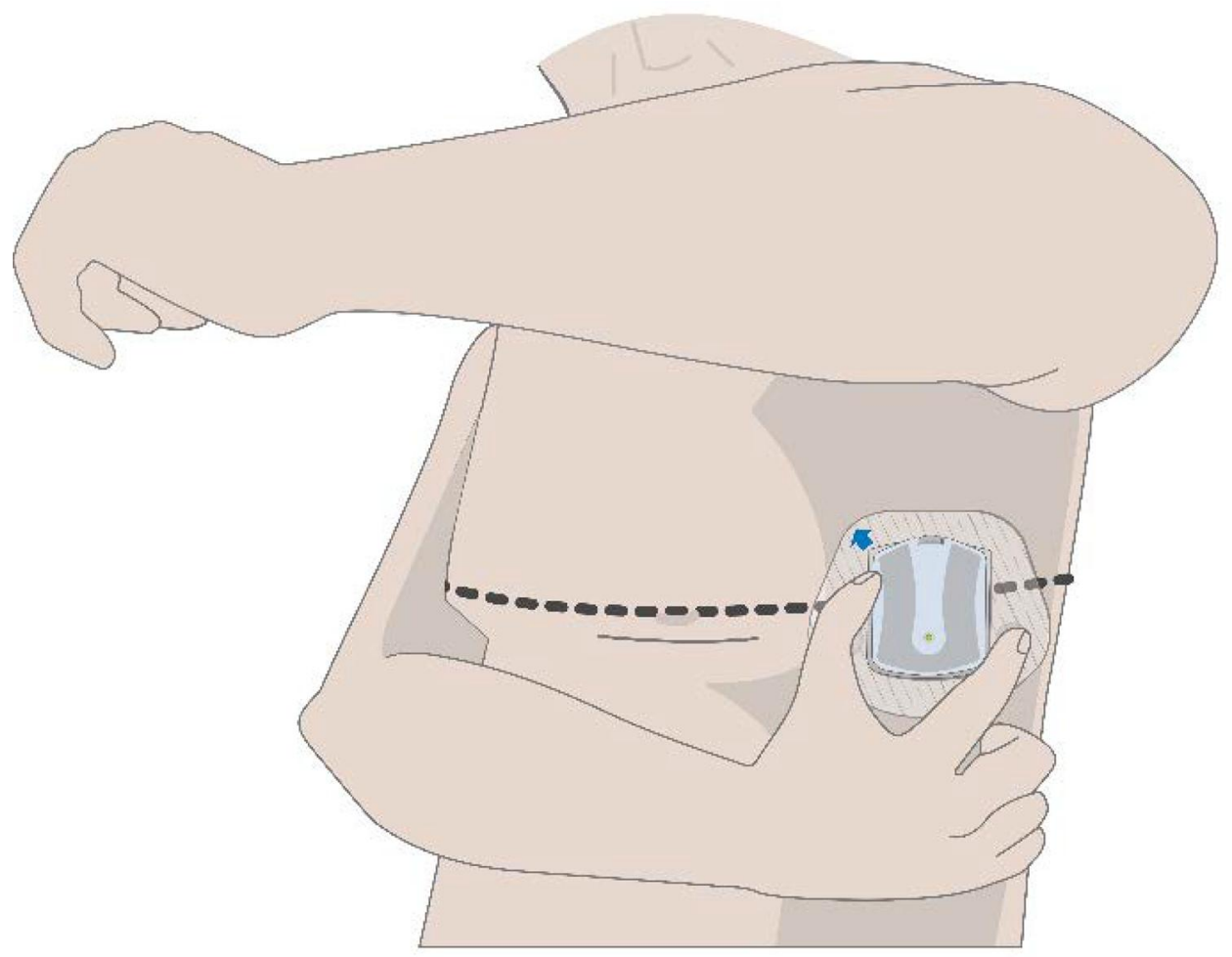

Figure I Illustration of the $\mu$ Cor device in the side location of a subject. The device emits radiofrequency signals which propagate through the lungs and are reflected back to the device. Changes in the reflected radar wave signal indicate changes in fluid accumulation.

hemodialysis session. During the course of the hemodialysis session, automated measurements of the UFV (in $\mathrm{mL}$ ) were obtained from the dialysis machine every 6 $\min ( \pm 1 \mathrm{~min})$. These UFV measurements were timesynchronized with the TFI readings within $1.5 \mathrm{~min}$.

After 15-min post-dialysis, the $\mu$ Cor device was removed and subject weight, blood pressure, and heart rate were recorded. The oral intake of any fluids or solids and the volume of intravenous (IV) fluids administered during the hemodialysis session were recorded. The volume of urine output during the hemodialysis session also was recorded. Adverse events were collected from the time of enrollment through the end of the study.

\section{Data Analysis}

Pearson's correlation coefficients were calculated for UFV and TFI for each individual subject. To determine the average correlation across all subjects, a Fisher's $z$ transformation was used because previous studies have shown that a simple arithmetic average of correlations leads to an underestimation of the population mean and hence a biased statistic. ${ }^{9,10}$ This underestimation occurs because the sampling distribution for correlation coefficients is skewed. To account for this bias, a Fisher Transformation (Eqn. 1) was used.

$$
z=0.5 \log e^{\left(\frac{1+r}{1-r}\right)}
$$

where $z$ is the Fisher transformed value of each subject's Pearson correlation coefficient, $r$.

An arithmetic average was performed on the $z$ values before back-transforming the averaged $\bar{z}$ value to give a mean value of $\bar{r}$ (Eqn. 2),

$$
\bar{r}=\frac{e^{2 \bar{z}-1}}{e^{2 \bar{z}+1}}
$$

Using this back-transformed value of $\bar{r}, 95 \%$ confidence intervals were calculated using the variance (Eqn. 3) derived from the Taylor Series expansion and Delta method.

$$
\begin{aligned}
\operatorname{Var}(\bar{r})= & \frac{1}{k^{2}} \sum_{i=1}^{i=k}(1-\bar{r}) \frac{1}{n_{i}-3} \\
& -\frac{\left[2\left(1-\bar{r}^{2}\right)-2(1+\bar{r})^{2}(1-\bar{r})\right]^{2}}{4\left(n_{i}-3\right)^{2}}
\end{aligned}
$$

where $k$ is the number of subjects, $\bar{r}$ is the Fisher backtransformed mean Pearson correlation coefficient (Eqn. 2), 
and $n_{i}$ is the number of paired data points (TFI and UFV) for each subject. Note, in order to calculate the variance, a minimum of four pairs of data points were required.

A responder analysis was performed to assess the variability of individual change in TFI by subject and to visualize absolute changes in TFI before and after dialysis by subject. The responder analysis calculated the percent reduction in TFI from baseline to completion of dialysis. A multivariate analysis was performed to determine whether BMI and NYHA class were significantly associated with a percent reduction in TFI before and after dialysis (R Core Team 2018). $P$ values less than 0.05 were determined to be statistically significant.

\section{Results}

The mean age (years) of the 20 enrolled subjects was 58 . Overall, subjects were mostly men $(80 \%)$ and Black (65.0\%). Subjects had a mean duration of hemodialysis of 7.5 years and $60 \%$ of the subjects had a history of heart failure. All demographics are shown in Table 1. The mean body mass index (BMI) of all subjects was 31 with a standard deviation of 9. There were no serious adverse events (SAEs) reported during the study. All AEs were anticipated and related to the dialysis procedure. There were no study device ( $\mu$ Cor system) issues related to AEs.

The mean volume of fluid removal was $3.63 \mathrm{~L}( \pm 0.88 \mathrm{~L})$. Individual Pearson correlation values of the subject's TFI to the subject's UFV are shown in Table 2. Of the 20 subjects, 14 subjects exhibited very strong correlations $(r>0.9), 3$ subjects showed a strong correlation $(0.7<r<0.9)$, and two subjects showed a poor correlation $(r<0.5)$. Data from subject (202) were not assessed as RF measurements of adequate quality could not be obtained. Figure 2 displays the relationship between TFI and UFV within each subject that had adequate quality measurements. The total number of valid TFI measurements taken for each subject is shown in the third column of Table 2 and ranges from 3 measurements to 35 measurements. One subject's correlation values (334) were excluded from the Fisher Transform analysis due to the requirement of at least 4 pairs of data for the Fisher Transform formula. For 18 subjects that had at least 4 pairs of data, the average correlation between fluid removal and TFI was $r=0.95$ CI [0.92-0.99].

The responder analysis, shown in Figure 3, demonstrated that all subjects experienced a reduction in TFI from baseline to the end of dialysis. The mean reduction of TFI was $5.5 \% \pm$ 3.8 with a maximum reduction of $13.9 \%$, and minimum reduction of $1.4 \%$. Subject BMI prior to UFV was investigated
Table I Demographic Data of the Subjects Enrolled

\begin{tabular}{|c|c|}
\hline Parameters & $\mathbf{N}=\mathbf{2 0}$ \\
\hline \multicolumn{2}{|l|}{ Age (years) } \\
\hline Mean (SD) & $58(9.5)$ \\
\hline Minimum, Maximum & $35-76$ \\
\hline \multicolumn{2}{|l|}{$\operatorname{Sex}(n[\%])$} \\
\hline Female & $6(30)$ \\
\hline Male & $14(70)$ \\
\hline \multicolumn{2}{|l|}{ Race (n [\%]) } \\
\hline American Indian/Alaska Native & $3(15)$ \\
\hline Black & $13(65)$ \\
\hline Caucasian & $3(15)$ \\
\hline Native Hawaiian or Other Pacific Islander & I (5) \\
\hline \multicolumn{2}{|l|}{ Ethnicity (n [\%]) } \\
\hline Hispanic or Latino & $2(10)$ \\
\hline Not Hispanic or Latino & $18(90)$ \\
\hline \multicolumn{2}{|l|}{ Baseline Body Mass Index $\left(\mathrm{kg} / \mathrm{m}^{2}\right)$} \\
\hline Mean (SD) & $31.3(8.8)$ \\
\hline Minimum, Maximum & $20-55.6$ \\
\hline \multicolumn{2}{|l|}{ Duration on Hemodialysis (years) } \\
\hline Mean (SD) & $7.5(8.7)$ \\
\hline Minimum, Maximum & $0.6-37$ \\
\hline \multicolumn{2}{|l|}{ Daily Urine Output (mL) } \\
\hline $\mathrm{n}$ & 17 \\
\hline Mean (SD) & $260(5 \mid 8)$ \\
\hline Minimum, Maximum & $0-2000$ \\
\hline \multicolumn{2}{|l|}{ History of Heart Failure (n [\%]) } \\
\hline NYHA Class (n [\%]) & $12(60)$ \\
\hline II & $10(50)$ \\
\hline III & $2(10)$ \\
\hline
\end{tabular}

as a potential influence in the responder analysis, but when subject BMI was compared to the subject's respective TFI percent change, a weak Pearson correlation value $(r)$ of 0.13 was observed. Of the 11 subjects with TFI data that had an NYHA classification $>0,6$ subjects had a TFI percent change of $0-5 \%, 4$ subjects had a TFI percent change between $5 \%$ and $10 \%$, and 1 subject had a TFI percent change greater than $10 \%$. Initial subject BMI and NYHA class were investigated as potential influencers on the TFI reduction. Multivariate regression analysis suggested that reduction in TFI from baseline to the end of dialysis was not significantly associated with neither BMI $(\mathrm{p}=0.58)$ nor NYHA $(\mathrm{p}=0.87)$.

\section{Discussion}

There was a strong correlation (0.95 CI [0.92-0.99]) of TFI and UFV when individual Pearson correlation 
Table 2 Individual Pearson $r$ Correlations of UFV vs TFI with Corresponding Number of Measurements. The Fisher's Mean $r$ with $95 \% \mathrm{Cl}$ is Reported

\begin{tabular}{|l|l|l|}
\hline Subject ID & $\begin{array}{l}\boldsymbol{r} \text { (Pearson's } \\
\text { Correlation) }\end{array}$ & $\begin{array}{l}\text { Number of TFI } \\
\text { Measurements }\end{array}$ \\
\hline 101 & -0.97 & 32 \\
103 & -0.84 & 5 \\
106 & -0.95 & 14 \\
107 & -0.84 & 16 \\
$1 \mathrm{II}$ & -0.94 & 22 \\
112 & -0.03 & 13 \\
133 & -0.99 & 32 \\
134 & -0.21 & 17 \\
135 & -1 & 29 \\
202 & $\mathrm{NA}$ & $\mathrm{NA}$ \\
203 & -0.99 & 26 \\
231 & -0.99 & 27 \\
232 & -0.94 & 29 \\
303 & -1 & 37 \\
304 & -0.92 & 31 \\
306 & -0.99 & 4 \\
308 & -0.99 & 34 \\
332 & -1 & 35 \\
334 & -0.95 & 3 \\
336 & -0.74 & 30 \\
\hline Fisher's Mean $r$ and & $0.95(0.92-0.99)$ & \\
$95 \%$ Cl & & \\
\hline & & \\
\hline
\end{tabular}

coefficients were averaged through the Fisher's $z$ transformation. The correlation demonstrated that the $\mu$ Cor system may have utility in tracking total body fluid in dialysis patients.

Some subjects' TFI responded more strongly to changes in UFV when compared to the TFI response observed in other subjects. We believe these differences in responses could be explained by variables that affect fluid removal in intravascular and interstitial compartments during dialysis. It is possible that for some subjects, even though the fluid is removed from the intravascular compartment, only a small portion of the total fluid is removed from the interstitial compartment during dialysis. ${ }^{11}$ Supporting evidence of this phenomenon was found in a hemodialysis study, where $31 \%$ of subjects following dialysis still had moderate to severe pulmonary congestion demonstrating that fluid removal is often not completely fulfilled. ${ }^{8}$ It is also possible that individuals with mild pulmonary congestion may experience a more dramatic change in TFI. These possibilities may explain the outcomes of the responder analysis shown in Figure 3 where some subjects exhibited more robust responses in TFI changes than other subjects. All responders however were downward trending.

The findings of the study have implications for the value derived from the patient perspective, both in fluid monitoring during dialysis and fluid monitoring within other disease states such as HF. When patients and clinicians are aware of fluid status, treatments can be tailored to the patient so that benefits are optimized and safety from harmful side effects is reduced. The design of the study device as an RF-based wearable patch also offers advantages compared to current fluid monitors as the device avoids the expertise needed to interpret LUS, and its RF-based measurement is differentiated from traditional bioimpedance monitors where measurements of whole-body fluid status become less sensitive to fluid in the trunk. ${ }^{12,13}$

Reliable remote monitoring of fluid status provides value to the patient as a safety/efficacy monitor in the home hemodialysis setting, a setting which is the focus of a recent initiative set by the US government to broaden home hemodialysis programs. ${ }^{14}$ Telemonitoring techniques and devices have recently been identified as innovative approaches for fluid monitoring in dialysis patients, with the potential to improve morbidity and mortality. ${ }^{15}$ Wearable devices also have the capability to expand upon a singular measurement such as radar or bioimpedance, and integrate multiple biologic signals to provide a comprehensive clinical picture. $^{7}$ Furthermore, all patients who have access to these data in the setting of home monitoring may become more involved in their care as the consequences of choices that affect the patient's health may be apparent before symptoms develop.

The study is limited in its scope of 20 hemodialysis subjects. Additionally, all subjects were undergoing chronic hemodialysis and patients new to hemodialysis may have exhibited different findings. The clinical indication for dialysis was unknown for all subjects, and differences in clinical background could have influenced the RF readings. There was a variable number of valid measurements among subjects as only those data points with predetermined signal quality were used for the analysis. The device is limited in its ability to estimate the dry weight of the subject due to the fact that the TFI reading is normalized. Furthermore, total fluid removal measured by UFV may not directly represent changes in the lung fluid captured by a TFI measurement taken in the left lung tissue. 

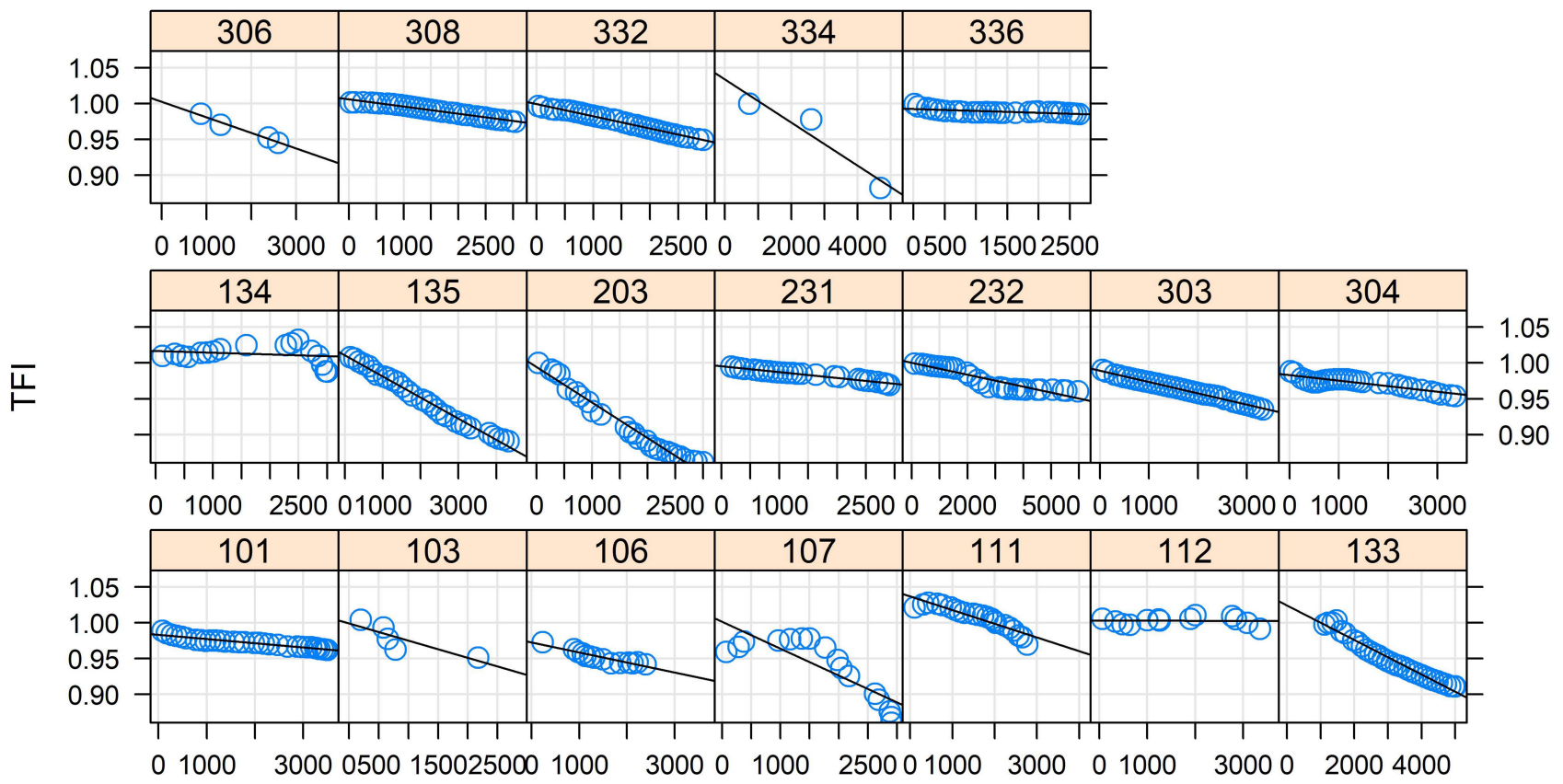

UFV

Figure 2 Individual plots by subject of TFI vs UFV ( $\mathrm{mL}$ ) are shown. The number above each plot corresponds to the individual subject study identification number.

\section{TFI Responder Assessment}

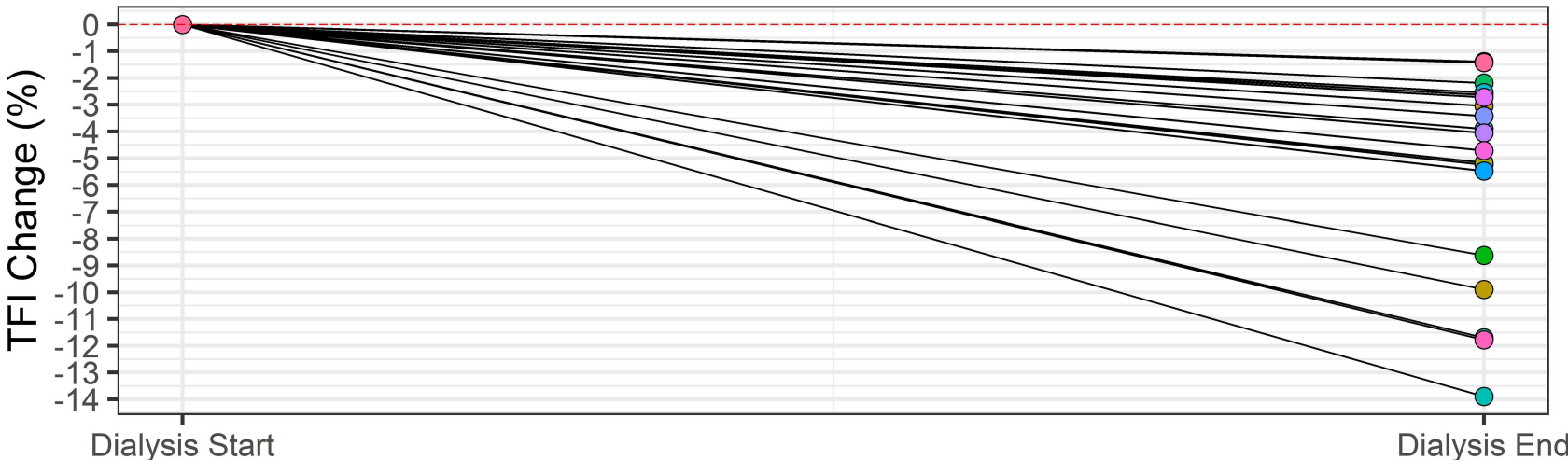

Figure 3 TFI Responder analysis showing percent change in TFI from beginning of dialysis to the end of dialysis. Some subjects experienced greater absolute TFI changes than other subjects.

\section{Conclusion}

The $\mu$ Cor system provides RF-based measurements of thoracic fluid which correlate well with total body fluid removal in a real-world setting. TFI measurements obtained by the $\mu$ Cor system may be useful in fluid management. Fluid management in dialysis patients serves as a potential model for the management of pulmonary congestion in a variety of clinical settings and conditions characterized by volume overload, including congestive HF. This novel wearable technology has the potential to empower end-stage kidney disease patients and those with $\mathrm{HF}$ to improve their clinical outcomes.

\section{Funding}

This trial was funded by ZOLL Medical Corporation.

\section{Disclosures}

MS and RP are employees of ZOLL. JC is a consultant for Achaogen, Akebia, Dynavax, GSK, and Relypsa; research support for Akebia, Astra Zeneca, Cara Therapeutics, 
Gilead, GSK, ZOLL; and is an employee of DaVita Clinical Research and a stockholder of DaVita Inc. $\mathrm{CH}$ is a consultant for Abbvie, Amgen, AstraZeneca, Corvidia, Diamedica, FibroGen, Janssen, NxStage, Pfizer, Relypsa, Sanifit, and University of Oxford; grant/research support for Amgen, Bristol-Myers Squibb, NIDDK/NIH, Relypsa, and University of British Columbia; author royalties from UptoDate; is an employee of Hennepin Healthcare and a stockholder for Boston Scientific, Bristol-Myers Squibb, General Electric, Johnson and Johnson, and Merck. The authors report no other conflicts of interest in this work.

\section{References}

1. Prowle JR, Jirwan CJ, Bellomo R. Fluid management for the prevention and attenuation of acute kidney injury. Nat Rev Nephrol. 2013;10:37-47.

2. Payen D, De Pont AC, Sakr Y, et al. A positive fluid balance is associated with a worse outcome in patients with acute renal failure. Crit Care. 2008;12(3):1-7. doi:10.1186/cc6916

3. Chawla LS, Herzog CA, Costanzo MR, et al. Proposal for a functional classification system of heart failure in patients with end-stage renal disease: proceedings of the Acute Dialysis Quality Initiative (ADQI) XI workgroup. $J \mathrm{Am}$ Coll Cardiol. 2014;63(13):1246-1252. doi:10.1016/j.jacc.2014.01.020

4. Rezaeieh SA, Zamani A, Bialkowski KS, Mahmoud A, Abbosh AM. Feasibility of using wideband microwave system for non- invasive detection and monitoring of pulmonary oedema. Nat Publ Gr. 2015; (August):1-11.

5. DeVore AD, Wosick J, Hernandez AF. The future of wearables in heart failure. JACC Hear Fail. 2019;7:11.
6. Efremov SM, Kuzkov VV, Fot EV, et al. Lung ultrasonography and cardiac surgery: a narrative review. J Cardiothorac Vasc Anesth. 2020;34.

7. Reljin N, Posada-Quintero HF, Eaton-Robb C, et al. Machine learning model based on transthoracic bioimpedance and heart rate variability for lung fluid accumulation detection: prospective clinical study. JMIR Med Informatics. 2020;8(8):e18715. doi:10.2196/18715

8. Mallamaci F, Benedetto FA, Tripepi R, et al. Detection of pulmonary congestion by chest ultrasound in dialysis patients. JACC Cardiovasc Imaging. 2010;3(6):586-594. doi:10.1016/j.jcmg.2010.02.005

9. Clayton Silver NP, Dunlap W. Averaging correlation coefficients: should Fisher's z transformation be used? J Appl Psychol. 1987;72 (1):146-148. doi:10.1037/0021-9010.72.1.146

10. Corey D, Dunlap W. Averaging correlations: expected values and bias in combined Pearson and Fisher's z transformation. J Gen Psychol. 1998;125:245-261. doi:10.1080/00221309809595548

11. Chase SC, Fermoyle CC, Wheatley CM, Schaefer JJ, Olson LJ, Johnson BD. The effect of diuresis on extravascular lung water and pulmonary function in acute decompensated heart failure. ESC Hear Fail. 2018;5(2):364-371. doi:10.1002/ehf2.12253

12. Bracco D, Thiébaud D, Chioléro RL, Landry M, Burckhardt P, Schutz Y. Segmental body composition assessed by bioelectrical impedance analysis and DEXA in humans. J Appl Physiol. 1996;81 (6):2580-2587. doi:10.1152/jappl.1996.81.6.2580

13. Kotanko P, Levin NW, Zhu F. Current state of bioimpedance technologies in dialysis. Nephrol Dial Transplant. 2008;23(3):808-812. doi:10.1093/ndt/gfm889

14. Executive Order on Advancing American Kidney Health. Available from: https://www.whitehouse.gov/presidential-actions/executiveorder-advancing-american-kidney-health/. Accessed October 30, 2020.

15. Chait Y, Derk G, Forfang D, et al. Fostering innovation in fluid management. Kidney Heal Initiat. 2019;(July):1-18.
Medical Devices: Evidence and Research

\section{Publish your work in this journal}

Medical Devices: Evidence and Research is an international, peerreviewed, open access journal that focuses on the evidence, technology, research, and expert opinion supporting the use and application of medical devices in the diagnosis, monitoring, treatment and management of clinical conditions and physiological processes. The identification of novel devices and optimal use of existing devices which will lead to improved clinical outcomes and more effective patient management and safety is a key feature of the journal. The manuscript management system is completely online and includes a very quick and fair peer-review system. Visit http:// www.dovepress.com/testimonials.php to read real quotes from published authors. 